02,08

\title{
Дефектонный вклад в высокотемпературную сверхпроводимость гидридов
}

\author{
(C) А.И. Морозов \\ Московский фризико-технический институт (государственный университет), \\ Долгопрудный, Россия \\ E-mail: mor-alexandr@yandex.ru
}

Поступила в Редакцию 21 февраля 2019 г.

В окончательной редакции 21 февраля 2019 г.

Принята к публикации 26 февраля 2019 г.

Высокое давление в сотни гигапаскалей, необходимое для получения высокотемпературных сверхпроводящих фаз ряда гидридов, вызывает сжатие кристаллической решетки, что, в свою очередь, ведет к экспоненциальному росту вероятности туннелирования атома водорода между эквивалентными междоузлиями. При низких температурах вакансии в заполненной при стехиометрии подрешетке водорода и атомы водорода в полностью свободной при стехиометрии подрешетке междоузлий являются квантовыми дефектами дефектонами. Получена оценка вклада дефектонов в сверхпроводимость гидридов и показано, что он может быть существенным как в случае свободных дефектонов, так и в случае дефектонов, кластеризовавшихся с образованием двухуровневых систем.

Ключевые слова: квантовые дефекты (дефектоны); сверхпроводимость; высокие давления; кластеризация; двухуровневые системы.

DOI: $10.21883 /$ FTT.2019.07.47830.385

\section{1. Введение}

После экспериментального обнаружения при давлении $155 \mathrm{GPa}$ высокотемпературной сверхпроводимости в соединении $\mathrm{H}_{3} \mathrm{~S}$ с критической температурой $T_{c}$, превосходящей $200 \mathrm{~K}[1]$, интерес к исследованию сверхпроводимости гидридов достиг рекордных высот. На основании компьютерного моделирования было предсказано существование целого ряда высокотемпературных сверхпроводящих гидридов при давлениях в сотни гигапаскалей (смотри обзор [2]). В основу теоретического описания сверхпроводимости гидридов положен электрон-фононный механизм [3-6].

Изотопы водорода в матрице металла являются практически единственными (за исключением гелия) атомами, квантовое туннелирование которых между эквивалентными междоузлиями экспериментально наблюдаемо. Такие квантовые дефекты в дальнейшем будем называть дефектонами.

Основополагающие проблемы квантовой диффузии были изучены в пионерской работе [7]. Авторы указали на тот факт, что квантовый дефект в идеальном кристалле в области низких температур делокализован и описывается блоховской волновой функцией. Подробное рассмотрение дефектонов в металлах и их вклада в сверхпроводимость впервые было проведено в работе [8].

Данная статья посвящена оценке туннельного матричного элемента атома водорода в сильно сжатых высокотемпературных сверхпроводящих гидридах и вклада электрон-дефектонного взаимодействия в сверхпроводимость.

\section{2. Дефектоны в гидриде металла}

Рассмотрим гидрид металла $\mathrm{MeH}_{x}$, близкий к стехиометрическому составу. При стехиометрическом составе и нулевой температуре квантовые дефекты отсутствуют, однако с ростом температуры возникают френкелевские пары квантовых дефектов: вакансия в подрешетке водорода, которая в основном состоянии была полностью заполненной, и атом водорода в подрешетке междоузлий, которая в основном состоянии не содержала атомов. Если концентрация водорода больше (меньше), чем стехиометрическая, то дефектоны - атомы водорода (вакансии) в указанных подрешетках существуют уже при нулевой температуре. Мы рассмотрим именно этот случай, считая концентрацию дефектонов не зависящей от температуры.

Поскольку высокое давление, необходимое для получения высокотемпературных сверхпроводящих фаз, уменьшает межузельное расстояние, от которого туннельный матричный элемент $t$ дефектона зависит экспоненциально, то в области высоких давлений можно ожидать высоких значений $t$. Для его оценки используем следующий простой алгоритм.

Здесь и в дальнейшем будем предполагать, что атом водорода находится на основном колебательном уровне в потенциальной яме, которой для водорода является междоузлие в матрице металла. Вследствие туннелирования атома между эквивалентными междоузлиями этот уровень порождает дефектонную зону. Величину 
Зависимость туннельного матричного элемента от междоузельного расстояния

\begin{tabular}{r|c|c}
\hline$t, \mathrm{~K}$ & $a$ & $d, \AA$ \\
\hline 100 & 5.42 & 0.87 \\
10 & 6.22 & 1.00 \\
1 & 6.92 & 1.11
\end{tabular}

туннельного матричного элемента можно оценить как

$$
t(a)=\operatorname{Ry} \int_{-\infty}^{+\infty} \psi_{0}\left(x-\frac{a}{2}\right) \psi_{0}\left(x+\frac{a}{2}\right) d x,
$$

где Ry - ридберговская энергия $13.6 \mathrm{eV}, a$ - обезразмеренное расстояние между положениями равновесия атома водорода в соседних междоузлиях

$$
a=d \sqrt{\frac{m \omega}{\hbar}},
$$

где $d$ - расстояние между положениями равновесия, $m$ - масса атома водорода, а $\omega-$ характерная частота оптических или локальных колебаний этого атома. Волновая функция $\psi_{0}(x)$ основного состояния гармонического осциллятора имеет вид

$$
\psi_{0}(x)=\frac{1}{\sqrt{\pi}} \exp \left(-\frac{x^{2}}{2}\right) .
$$

Легко видеть, что

$$
t(a)=\operatorname{Ry} \exp \left(-\frac{a^{2}}{4}\right) .
$$

Используя для $\omega$ величину $\hbar \omega=160 \mathrm{meV}$, выбранную на основе фононных спектров, рассчитанных в работе [6], получаем значения $t$, приведенные в таблице.

Проведенное для целого ряда высокотемпературных сверхпроводящих гидридов численное моделирование показывает, что во многих сверхпроводящих фазах величина $d \lesssim 1 \AA$ [9-11], например, $0.841 \AA$ в $\mathrm{SnH}_{4}$ [11].

Следует отметить, что ширина дефектонной зоны $\varepsilon$ в $2 z$ раз превосходит $t(z-$ число ближайших эквивалентных междоузлий).

В нормальном металле основной вклад в затухание дефектонов вносит их рассеяние на электронах [8], а фононный вклад в затухание, рассмотренный в работе [7], становится существенным только в сверхпроводящей фазе при $T \ll T_{c}$.

Характерное время рассеяния дефектонов на электронах $\tau_{e}$ равно [8]

$$
\tau_{e}^{-1}=\frac{4 \pi g T}{\hbar\left[1+\exp \left(\frac{\Delta}{T}\right)\right]},
$$

где $\Delta-$ сверхпроводящая щель в спектре электронных возбуждений. В нормальной фазе

$$
\tau_{e}^{-1}=2 \pi g T / \hbar
$$

Параметр $g$ равен

$$
g=2 \int \frac{d^{2} \mathbf{k} d^{2} \mathbf{k}^{\prime}\left|V_{0}\left(\mathbf{k}-\mathbf{k}^{\prime}\right)\right|^{2}}{(2 \pi)^{6}|\nabla E(\mathbf{k})|\left|\nabla E\left(\mathbf{k}^{\prime}\right)\right|},
$$

интегрирование происходит по электронной поверхности Ферми, $E(\mathbf{k})$ - закон дисперсии электронов, $V_{0}(\mathbf{q})$ - затравочная амплитуда рассеяния электрона на дефектоне. Выражения (5), (6) справедливы при $\max (\Delta, T) \gg \varepsilon$. В нормальной фазе при $T<\varepsilon$ в правой части выражения (6) возникает дополнительный множитель $T / \varepsilon[12]$.

Предшествующее рассмотрение проведено в предположении о зонном характере движения дефектона, которое справедливо, если его энергия намного превосходит величину $\hbar \tau_{e}^{-1}$. С повышением температуры движение дефектона приобретает прыжковый характер, последовательное описание которого отсутствует.

При исследовании электрон-дефектонного взаимодействия нельзя ограничиться борновским приближением, так как вследствие наличия резкого края электронного ферми-заполнения, возникают инфракрасные (ИК) расходимости, на которые обратил внимание Кондо [13]. По аналогии с расчетом аномалий поглощения и испускания рентгеновских лучей в металлах [14-16] был проведен последовательный учет ИК перенормировок параметров дефектона, результаты которого изложены в обзоре [17]. Однако на этом трудности, связанные с описанием дефектонов, не заканчиваются.

\section{3. Кластеризация квантовых дефектов}

Описание дефектонов осложняется тем фактом, что подвижные дефекты в металле кластеризуются с понижением температуры [18]. Кластеризация обусловлена тем, что дальнодействующая часть взаимодействия между точечными дефектами в металле является знакопеременной. Она складывается из упругого взаимодействия $W_{\text {elas }}$ и взаимодействия через фриделевские осцилляции электронной плотности $W_{\mathrm{el}}$

$$
W(\mathbf{R})=W_{\text {elas }}(\mathbf{R})+W_{\text {el }}(\mathbf{R}),
$$

где $\mathbf{R}$ - радиус-вектор, соединяющий дефекты.

Упругое взаимодействие имеет вид [19]:

$$
W_{\text {elas }}(\mathbf{R})=W_{1}(\mathbf{n}) \frac{\Omega}{R^{3}},
$$

где $\Omega$ - объем элементарной ячейки, $\mathbf{n}=\mathbf{R} / R$, a $W_{1}(\mathbf{n})$ принимает положительные и отрицательные значения в зависимости от ориентации вектора $\mathbf{n}$ относительно кристаллографических осей. Значение $W_{1}(\mathbf{n})$ изменяется от $1 \mathrm{eV}$ для тяжелых примесей внедрения до $10^{-2} \mathrm{eV}$ для случая двух атомов водорода в металле [20].

Взаимодействие через фриделевские осцилляции электронной плотности, которое для случая сферической 
поверхности Ферми радиуса $k_{\mathrm{F}}$ имеет вид [21]

$$
W_{\mathrm{el}}(\mathbf{R})=W_{2} \frac{\Omega}{R^{3}} \cos \left(2 k_{\mathrm{F}} R\right),
$$

где $W_{2}=$ const $\sim 10^{-2} \mathrm{eV}$, играет не меньшую роль, чем упругое взаимодействие, и так же спадает с расстоянием между дефектами.

В силу знакопеременности $W(\mathbf{R})$ имеется целый набор связанных состояний двух дефектонов или дефектона и тяжелой „замороженной“ примеси. Наибольшей энергии связи $W_{0}$ соответствует $R \sim d$. Короткодействующая часть взаимодействия между дефектами может изменить знак энергии взаимодействия только для нескольких самых малых значений $\mathbf{R}$. Поэтому все подвижные точечные дефекты в металле (и нейтральные примеси в диэлектрике) кластеризуются с понижением температуры $[18,22]$. Характерная температура кластеризации в области низкой концентрации дефектонов $x \ll 1$ равна по порядку величины

$$
T_{\mathrm{cl}} \approx W_{0} /|\ln x|
$$

Часто в результате кластеризации вместо крупномасштабного расслоения на фазы с высокой и низкой концентрацией водорода возникает метастабильное состояние с кластерами из конечного числа частиц.

В кристалле, в котором концентрация тяжелых „замороженных“ примесей сравнима с концентрацией дефектонов, возникают кластеры из легкой и тяжелой примеси. В ряде случаев атом водорода занимает одно из двух эквивалентных, с точки зрения энергии связи с тяжелой примесью, положений равновесия в двух соседних междоузлиях. Вследствие его туннелирования между ними возникает двухуровневая система (ДУС). Примером возникновения ДУС является захват водорода тяжелыми примесями кислорода, азота или углерода в ниобии. Экспериментально измеренное значение туннельного матричного элемента $t \sim 90 \mu \mathrm{eV}$ [23] всего в несколько раз отличается от оценки $t \sim 27 \mu \mathrm{eV}$, полученной по формуле (4).

Взаимное влияние ДУС приводит к нарушению эквивалентности двух минимумов потенциального рельефа ДУС и возникновению лоренцевского распределения разности энергий указанных минимумов с шириной $\delta=c W_{0}[24]$, где $c$ - концентрация ДУС.

Так же как и взаимодействие со свободными дефектонами, взаимодействие электронов с ДУС приводит к куперовскому спариванию и дает вклад в сверхпроводимость [25,26].

\section{4. Вклад свободных дефектонов в сверхпроводимость}

Последовательное описание вклада дефектонов (как свободных, так и ДУС) в сверхпроводимость с учетом ИК-перенормировок было проведено в работе [27]. Он
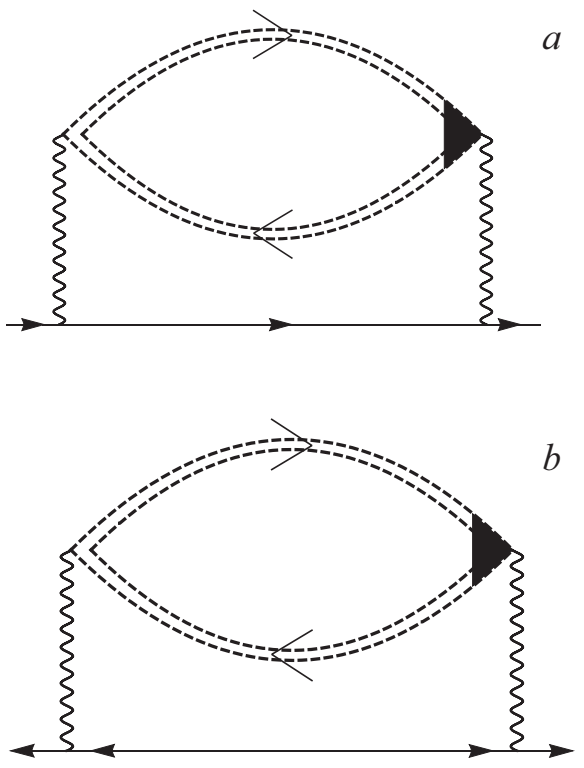

Диаграммы для неупругого вклада дефектонов в нормальную $(a)$ и аномальную $(b)$ собственно-энергетические части электронной функции Грина. Двойная пунктирная линия перенормированная функция Грина дефектона, сплошная линия - нормальная $(a)$ и аномальная $(b)$ функции Грина электрона, волнистая линия - затравочная амплитуда рассеяния электрона на дефектоне, волнистая линия с черным треугольником - полная амплитуда рассеяния электрона на дефектоне.

обусловлен неупругим рассеянием электронов на дефектонах. Соответствующие диаграммы для нормальной и аномальной собственно-энергетических частей электронной функции Грина изображены на рисунке. В случае, когда сверхпроводящая щель одинакова во всех точках поверхности Ферми (мы ограничимся этим случаем), упругое рассеяние на дефектах не влияет на сверхпроводимость. Общий вид уравнений Элиашберга [28] при наличии электрон-дефектонного взаимодействия приведен в работе [27].

Если предполагать, что основной вклад в сверхпроводимость дает электрон-дефектонное взаимодействие и критическая температура $T_{\mathrm{c}} \gg T_{\mathrm{cl}}$ (этот случай в [27] не рассматривался), то по аналогии с рассмотренным в ней случаем ДУС в приближении слабой связи $\left(\lambda_{d} \ll 1\right)$ получаем

$$
T_{\mathrm{c}} \approx \varepsilon \exp \left(-\frac{1+\lambda_{d}}{\lambda_{d}}\right),
$$

где

$$
\lambda_{d} \approx \frac{x g E_{0}}{\varepsilon}
$$

a $E_{0}$ - характерная ширина электронной зоны проводимости.

На границе применимости оценка (12) дает максимально достижимое на основе дефектонного механизма сверхпроводимости значение $T_{\mathrm{c}} \approx \varepsilon$. Именно этот факт делает актуальным данный механизм в фазах высокого давления. 
Если же основной вклад в сверхпроводимость дает электрон-фононный механизм, то при $T_{\mathrm{cl}} \ll T \ll T_{\mathrm{c}}$ малая добавка $\Delta_{1}$, обусловленная электрон-дефектонным взаимодействием, к сверхпроводящей щели $\Delta_{0}$ в спектре электронных возбуждений равна

$$
\Delta_{1} \approx \frac{x g \varepsilon E_{0}}{\Delta_{0}}
$$

и может достигать заметной величины. Для $x \sim 0.01$, $g \sim 0.1, \Delta_{0} \sim 200 \mathrm{~K}, E_{0} \sim 5 \cdot 10^{4} \mathrm{~K}$ и $\varepsilon \sim 50 \mathrm{~K}$ дефектонная поправка составляет величину порядка $6 \%$.

\section{5. Вклад ДУС в сверхпроводимость}

Если вклад ДУС в сверхпроводящее спаривание является определяющим, то, согласно [27], критическая температура сверхпроводящего перехода дается формулой

$$
T_{\mathrm{c}} \approx t \exp \left(-\frac{1+\lambda_{d}}{\lambda_{d}}\right),
$$

где

$$
\lambda_{d} \approx \frac{\operatorname{cg} E_{0}}{\max (t, \delta)} .
$$

При $c>c_{0} \approx t / W_{0}$ дальнейший рост концентрации ДУС не ведет к росту $\lambda_{d}$ и $T_{\mathrm{c}}$.

Если же вклад ДУС является дополнением к электронфононной сверхпроводимости, то при $c<c_{0}$ и $T \ll T_{\mathrm{c}}$

$$
\Delta_{1} \approx \frac{\operatorname{cgt} E_{0}}{\Delta_{0}} .
$$

Для $c \sim 0.01, g \sim 0.1, \Delta_{0} \sim 200 \mathrm{~K}, E_{0} \sim 5 \cdot 10^{4} \mathrm{~K}$ и $t \sim 10 \mathrm{~K}$ дефектонная поправка составляет величину порядка $1 \%$.

\section{6. Выводы}

1, Использование давлений в сотни гигапаскалей для получения высокотемпературных сверхпроводящих гидридов приводит к сжатию кристаллической решетки и экспоненциальному росту матричного элемента, описывающего туннелирование атома водорода между соседними междоузлиями.

2. В области низких температур может иметь место зонное движение квантовых дефектов - дефектонов. Дефектонами являются вакансии в полностью заполненной при нулевой температуре и стехиометрическом составе подрешетке водорода, а также атомы водорода в полностью свободной при этих же условиях подрешетке междоузлий.

3. С понижением температуры неизбежно происходит кластеризация дефектонов. В ряде случаев при этом возникают ДУС.

4. Как свободные дефектоны, так и ДУС вносят вклад в куперовское спаривание электронов, который может стать определяющим при больших ширинах дефектонной зоны (больших значениях туннельного матричного элемента ДУС).

\section{Список литературы}

[1] A.P. Drozdov, M.I. Eremets, I.A. Troyan, V. Ksenofontov, S.I. Shylin. Nature 525, 73 (2015).

[2] D. Duan, Y. Liu, Y. Ma, Z. Shao, B. Liu, T. Cui. Nature Sci. Rev. 4, 121 (2017).

[3] W. Sano, T. Koretsune, T. Tadano, R. Akashi, R. Arita. Phys. Rev. B 93, 094525 (2016).

[4] Q. Zhuang, X. Jin, T. Cui, D. Zhang, Y. Li, X. Li, K. Bao, B. Liu. Phys. Rev. B 98, 024514 (2018).

[5] L.P. Gor'kov, V.Z. Kresin. Rev. Mod. Phys. 90, 011001 (2018).

[6] К.С. Гришаков, Н.Н. Дегтяренко, Е.А. Мазур. ЖЭТФ 155, $116(2019)$

[7] А.Ф. Андреев, И.М. Лифшиц. ЖЭТФ 56, 2057 (1969).

[8] А.И. Морозов. ЖЭТФ 77, 1471 (1979).

[9] Y. Li, J. Hao, H. Liu, J.S. Tse, Y. Wang, Y. Ma. Sci. Rep. 5, 09948 (2015).

[10] X. Feng, J. Zhang, G. Gao, H. Liu, H. Wang. RSC Adv. 5, 59292 (2015).

[11] K. Tanaka, J.S. Tse, H. Liu. Phys. Rev. B 96, 100502(R) (2017).

[12] A.I. Morosov, A.S. Sigov. Solid State Commun. 53, 31 (1985).

[13] J. Kondo. Phys. B 123, 175 (1984).

[14] B. Roulet, J. Gavoret, P. Nozieres. Phys. Rev. 178, 1072 (1969).

[15] P. Nozieres, J. Gavoret, B. Roulet. Phys. Rev. 178, 1084 (1969).

[16] P. Nozieres, C.T. De Dominicis. Phys. Rev. 178, 1097 (1969).

[17] А.И. Морозов, А.С. Сигов. УФН 164, 243 (1994).

[18] А.И. Морозов, А.С. Сигов. ЖЭТФ 95, 170 (1989).

[19] Водород в металлах / Под ред. Г. Алефельда, И. Фелькля. Мир, М. (1969).

[20] В.А. Соменков, С.Ш. Шильштейн. Фазовые превращения водорода в металлах. ИАЭ, М. (1979). 81 с.

[21] У. Харрисон. Теория твердого тела. Мир, М. (1972). 616 с.

[22] A.I. Morosov, A.S. Sigov. Solid State Commun. 67, 841 (1988).

[23] D. Neumaier, H. Steinbinder, H. Wipf, G. Blank, Z. Kearley. Phys. B 76, 359 (1989).

[24] А.И. Морозов, А.С. Сигов. ЖЭТФ 98, 1454 (1990).

[25] А.П. Жернов. ФММ 67, 655 (1989).

[26] В.П. Минеев. Письма в ЖЭТФ 51, 399 (1990).

[27] А.И. Морозов, А.С. Сигов. ЖЭТФ 101, 919 (1992).

[28] Г.М. Элиашберг. ЖЭТФ 38, 966 (1960); ЖЭТФ 339, 1437 (1960).

Редактор Ю.Э. Китаев 